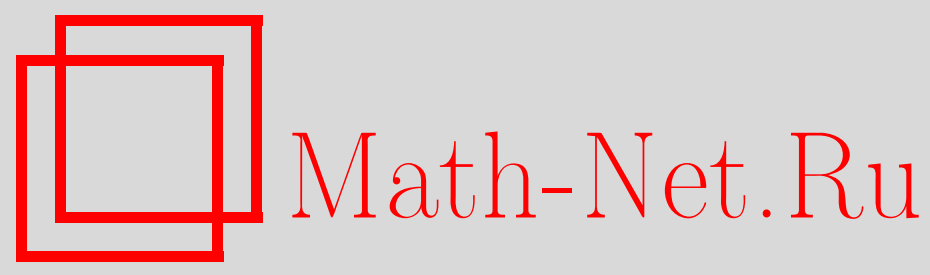

К. Д. Протасова, Уравновешенные разбиения графов, $M a-$ тем. заметки, 2006, том 79, выпуск 1, 127-133

DOI: https://doi.org/10.4213/mzm2681

Использование Общероссийского математического портала Math-Net.Ru подразумевает, что вы прочитали и согласны с пользовательским соглашением http://www . mathnet.ru/rus/agreement

Параметры загрузки:

IP: 18.208 .226 .222

26 апреля 2023 г., 14:22:54

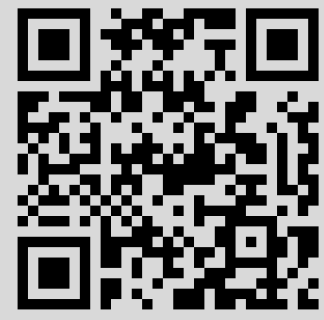


УДК 519.1

\section{УРАВНОВЕШЕННЫЕ РАЗБИЕНИЯ ГРАФОВ}

\section{К. Д. Протасова}

Доказано, что множество вершин $\mathscr{V},|\mathscr{V}|=r k$, связного граффа $G$ можно разбить на $r$ подмножеств, равных по числу элементов, так, что расстояние от любой вершины графа $G$ до каждого подмножества разбиения не превосходит $r$.

Библиограбия: 2 названия.

Рассмотрим конечный связный неориентированный граф $\mathrm{Gr}=(\mathscr{V}, E)$ с множеством вершин $\mathscr{V}$ и множеством ребер $E$. Для произвольных двух вершин $x, y \in \mathscr{V}$ обозначим через $d(x, y)$ длину кратчайшего пути от $x$ к $y$. Для произвольных вершины $x \in \mathcal{V}$, подмножества $A \subseteq \mathscr{V}$ и неотрицательного целого числа $m$ положим

$$
B(x, m)=\{y \in \mathscr{V}: d(x, y) \leqslant m\}, \quad B(A, m)=\bigcup_{a \in A} B(a, m)
$$

Индексом непустого подмножества $A \subseteq \mathscr{V}$ назовем наименьшее неотрицательное целое число $m$ такое, что $\mathscr{V}=B(A, m)$. Индекс подмножества $A$ обозначим ind $A$.

$P a c c m o я н и е ~ \operatorname{dist}(A, B)$ между непустыми подмножествами $A, B$ множества вершин $\mathscr{V}$ определяется формулой

$$
\operatorname{dist}(A, B)=\max \left\{\max _{a \in A} \min _{b \in B} d(a, b), \max _{b \in B} \min _{a \in A} d(b, a)\right\} .
$$

Отметим, что ind $A=\operatorname{dist}(\mathscr{V}, A)$ для любого непустого подмножества $A \subseteq \mathscr{V}$.

Индексом разбиения множества вершин $\mathscr{V}$ на непустые подмножества назовем максимальньй индекс подмножеств разбиения.

Разбиение конечного множества $X,|X|=n$, на $r$ подмножеств $(1 \leqslant r \leqslant n, n=r s+t$, $0 \leqslant t<r)$ назовем уравновешенным, если существует такая нумерация $X_{1}, X_{2}, \ldots, X_{r}$ подмножеств разбиения, для которой

$$
\left|X_{1}\right|=\left|X_{2}\right|=\cdots=\left|X_{t}\right|=s+1, \quad\left|X_{t+1}\right|=\left|X_{t+2}\right|=\cdots=\left|X_{r}\right|=s
$$

В частности, если $r$ - делитель $n$, то уравновешенное разбиение $X$ - это разбиение $X$ на $r$ частей, равных по числу элементов.

Цель заметки - доказать следующие три теоремы. 
ТЕоремА 1. Для любых натуральных чисел $r, n, r \leqslant n, u$ любого связного графа $\mathrm{Gr}=(\mathscr{V}, E),|\mathscr{V}|=n$, существует разбиение индекса $\leqslant r-1$ множества вериин $\mathscr{V}$ на $r$ подмножеств.

ТЕОрема 2. Для любых натуральных чисел $r, n, r \leqslant n, u$ любого связного графа $\mathrm{Gr}=(\mathscr{V}, E),|\mathscr{V}|=n$, существует уравновешенное разбиение индекса $\leqslant r$ множества вершин $\mathcal{V}$ на $r$ подмножеств.

ТЕорема 3. Пусть $r, n$ - натуральные числа, $r$-делитель $n$. Для любого связного графа $G(\mathscr{V}, E),|\mathscr{V}|=n$, существует уравновешенное разбиение $\mathscr{V}=\mathscr{V}_{1} \cup \mathscr{V}_{2} \cup$ $\cdots \cup \mathscr{V}_{r}$ maкое, чmo

$\operatorname{dist}\left(\mathscr{V}_{1}, \mathscr{V}_{2}\right) \leqslant 3, \quad \operatorname{dist}\left(\mathscr{V}_{2}, \mathscr{V}_{3}\right) \leqslant 3, \quad \ldots, \quad \operatorname{dist}\left(\mathscr{V}_{r-1}, \mathscr{V}_{r}\right) \leqslant 3, \quad \operatorname{dist}\left(\mathscr{V}_{r}, \mathscr{V}_{1}\right) \leqslant 3$

Для доказательства этих теорем удобно перейти к хроматической терминологии. Раскраска множества $X$ в $r$ цветов - это произвольное отображение "на" $\chi: X \rightarrow\{1$, $2, \ldots, r\}$. Каждая такая раскраска определяет разбиение

$$
\chi^{-1}(1) \cup \chi^{-1}(2) \cup \cdots \cup \chi^{-1}(r)
$$

множества $X$ на непустые подмножества. Наоборот, каждое разбиение $X=X_{1} \cup$ $X_{2} \cup \cdots \cup X_{r}$ множества $X$ на непустые подмножества порождается раскраской $\chi$, которая определяется правилом $\chi(x)=k$ тогда и только тогда, когда $x \in X_{k}$. Раскраску $\chi: X \rightarrow\{1,2, \ldots, r\}$ назовем уравновешенной, если разбиение

$$
X=\chi^{-1}(1) \cup \chi^{-1}(2) \cup \cdots \cup \chi^{-1}(r)
$$

является уравновешенным.

Разбиение множества вершин графа $G(\mathscr{V}, E)$ на $r$ подмножеств имеет индекс $\leqslant m$ тогда и только тогда, когда при соответствующей этому разбиению раскраске $\chi: \mathcal{V} \rightarrow$ $\{1,2, \ldots, r\}$ каждый шар $B(x, m), x \in \mathcal{V}$, содержит точки всех $r$ цветов. Индексом раскраски назьвается индекс соответствующего разбиения.

ДОКАЗАТЕЛЬСТВО ТЕОРЕМЫ 1. Индукцией по числу $n$ покажем, что существует pacкраска $\chi: \mathscr{V} \rightarrow\{1,2, \ldots, r\}$ такая, что $|\chi(B(x, k))| \geqslant k+1$ для всех $x \in \mathscr{V}, k \in$ $\{0,1, \ldots, r-1\}$. Заключение теоремы следует из этого утверждения при $k=r-1$.

Заменяя граф $G(\mathscr{V}, E)$ его остовом, можно считать, что $G(\mathscr{V}, E)$ является деревом. Для $n=1$ утверждение очевидно. Если $r=n$, то можно взять любую раскраску $\chi: \mathscr{V} \rightarrow\{1,2, \ldots, r\}$. Предположим, что $r<n, n>1$ и зафиксируем любую концевую вершину $y$ графа $G(\mathscr{V}, E)$. Тогда $B(y, 1)=\{y, z\}$, где $z$ - единственная смежная с $y$ вершина дерева. Рассмотрим граф $G_{1}\left(\mathscr{V}_{1}, E_{1}\right)$, где $\mathscr{V}_{1}=\mathscr{V} \backslash\{y\}, E_{1}=E \backslash\{(y, z)\}$. Обозначим через $B_{1}(x, k)$ шар радиуса $k$ в графе $G_{1}\left(\mathscr{V}_{1}, E_{1}\right)$ с центром в точке $x \in \mathscr{V}_{1}$. Так как граф $G_{1}\left(\mathscr{V}_{1}, E_{1}\right)$ связен и $\left|\mathscr{V}_{1}\right|=n-1$, то по предположению индукции существует раскраска $\chi_{1}: \mathscr{V}_{1} \rightarrow\{1,2, \ldots, r\}$ такая, что

$$
\left|\chi_{1}\left(B_{1}(x, k)\right)\right| \geqslant k+1
$$

для всех $x \in \mathscr{V}_{1}, k \in\{0,1, \ldots, r-1\}$. Заметим, что $\left|\chi_{1}(B(z, 0))\right|=1$ и выберем максимальное число $m \in\{0,1, \ldots, r-2\}$, для которого $\left|\chi_{1}\left(B_{1}(z, m)\right)\right|=m+1$. Далеевыберем 
произвольное число $s \in\{1,2, \ldots, r\}$ такое, что $s \notin \chi_{1}(B(z, m))$. Положим $\chi(y)=s$ и $\chi(x)=\chi_{1}(x)$ для всех $x \in \mathscr{V}_{1}$. Так как $B(z, k) \subseteq B(y, k+1)$, то $|\chi(B(y, k))| \geqslant k+1$ для всех $k \in\{0,1, \ldots, r-1\}$.

Доказательству теоремы 2 предпошлем пять лемм с дополнительными определениями.

Конечное дерево $G(\mathcal{V}, E),|\mathscr{V}|>2$, называется звездой с центром $x \in \mathscr{V}$, если $x$ не является концевой вершиной дерева и любые два кратчайшие пути от $x$ к различным конщевым вершинам не имеют общих ребер. Каждый такой путь $x=x_{0}, x_{1}, \ldots, x_{k}$ определяет луи звезды с множеством вершин $\left\{x_{0}, x_{1}, \ldots, x_{k}\right\}$ и множеством ребер $\left\{\left(x_{0}, x_{1}\right)\right.$, $\left.\left(x_{1}, x_{2}\right), \ldots,\left(x_{k-1}, x_{k}\right)\right\}$. Число $k$ назьвается длиной луча, а $x_{0}$ и $x_{k}$ - его началом и концом. Таким образом, каждая звезда является объединением $\geqslant 2$ лучей, исходящих из центра $x$. Радиусом звезды называется максимальная длина его лучей.

ЛЕмма 1. Пусть $r$ - натуральное число, $r>2, \operatorname{Gr}(V, E)$ - звезда с иентром $x$ и тремя лучами $R_{1}, R_{2}, R_{3}$ длины $r_{1}, r_{2}, r_{3}$, причем $r / 2 \leqslant r_{i} \leqslant r-1, i \in\{1,2,3\}$. Тогда существует уравновешенная $r$-раскраска индекса $\leqslant r$ мнохсества $\mathscr{V}$ такая, что на расстоянии $\leqslant r / 2$ от иентра имеются вершины всех $r$ иветов.

ДокАЗАТЕЛЬСТво. Пусть для определенности $r_{1} \geqslant r_{2} \geqslant r_{3}$. Запишем вершины лучей $R_{1}, R_{2}, R_{3}$ в порядке их расположения от начала к концам

$$
\left\{x, x_{1}, x_{2}, \ldots, x_{r_{1}}\right\}, \quad\left\{x, y_{1}, y_{2}, \ldots, y_{r_{2}}\right\}, \quad\left\{x, z_{1}, z_{2}, \ldots, z_{r_{3}}\right\}
$$

Рассмотрим последовательность вершин

$$
x_{r_{1}}, x_{r_{1}-1}, \ldots, x_{2}, x_{1}, x, z_{1}, z_{2}, \ldots, z_{r-r_{1}-1}
$$

и окрасим их цветами $1,2, \ldots, r$ слева направо.

Рассмотрим последовательность вершин $y_{1}, y_{2}, \ldots, y_{r_{2}}$ и окрасим их цветами 1,2 , $\ldots, r_{2}$ слева направо.

Отметим три наблюдения:

1) шар радиуса $r$ с центром $x_{r_{1}}$ содержит вершины всех $r$ цветов;

2) шар радиуса $r$ с центром $y_{r_{2}}$ содержит вершины всех $r$ цветов;

3) на расстоянии $\leqslant r / 2$ от центра звезды содержатся вершины всех $r$ цветов.

Таким образом, наша задача состоит лиш в том, чтобы докрасить уравновешенно вершины луча $R_{3}$ так, чтобы шар радиуса $r$ с центром $z_{r_{3}}$ также содержал вершины всех $r$ цветов.

Подсчитаем число $k$ неокрашенньх вершин:

$$
k=r_{3}-r+r_{1}+1
$$

Подсчитаем число $m$ окрашенных вершин в шаре радиуса $r$ с центром $z_{r_{3}}$ :

$$
m=\left(r-r_{3}\right)+\left(r-r_{3}\right)+\left(r-r_{1}\right)=3 r-2 r_{3}-r_{1} \text {. }
$$

Если $m \geqslant r$, то шар $B\left(r_{3}, r\right)$ содержит вершины всех $r$ цветов и мы можем докрасить вершины луча $R_{3}$ уравновешенно произвольным образом. 
Пусть $m<r$. Отметим, что каждый цвет $1,2, \ldots, r_{2}$ мы уже использовали дважды. Поскольку $r_{3} \leqslant r_{2}$,

$$
(r-m)+\left(r-r_{2}\right)=2 r_{3}+r_{1}-2 r+r-r_{2}=2 r_{3}+r_{1}-r_{2}-r \leqslant k=r_{3}-r+r_{1}+1 .
$$

Выберем $r-r_{2}$ неокрашенные вершины луча $R_{3}$ и окрасим их цветами $r_{2}+1, \ldots, r$. После этого каждьй из цветов $1,2, \ldots, r$ мы использовали дважды. Так как $r-m \leqslant$ $k-\left(r-r_{2}\right)$, мы имеем еше по меньшей мере $r-m$ неокрашенных вершин. Раскрасим их разньми цветами так, чтобы шар $B\left(z_{r_{3}}, r\right)$ содержал вершины всех $r$ цветов. Продолжим эту раскраску уравновешенно на множество вершин $V$.

ЛЕмма 2. Пусть $r$ - натуральное число $\geqslant 2, G(\mathscr{V}, E)$ - звезда с иентром $x$ радиуса $\leqslant r-1$, которая содержит не менее двух лучей длинь $\geqslant r / 2$. Тогда существует уравновешенная $r$-раскраска индекса $\leqslant r$ множества $\mathscr{V}$.

ДокАЗАТЕЛЬСТво. Пусть $R_{1}, R_{2}, \ldots, R_{t}$ - лучи длины $\geqslant r / 2, R_{t+1}, R_{t+2}, \ldots, R_{s}-$ лучи длины $<r / 2=R_{1} \cup R_{2} \cup \cdots \cup R_{t} \cup \cdots \cup R_{s}$. Предлагаемые способы раскраски зависят от четности числа $t$.

Если $t$ четно, занумеруем вершины графа $R_{1} \cup R_{2}$ последовательными натуральньми числами в порядке движения от конца луча $R_{1}$ к концу луча $R_{2}$. Продолжим нумерацию таким же образом на вершины графа $R_{3} \cup R_{4}$, пропуская центр $x$, и т.д. Начальным отрезком натурального ряда занумерованы вершины графра $R_{1} \cup R_{2} \cup \cdots \cup R_{t}$. Продолжая эту нумерацию произвольньм образом на остальные вершины звезды, получим упорядочение $x_{1}, x_{2}, \ldots, x_{n}$ множества $\mathscr{V}$. Окрасим вершины $x_{1}, x_{2}, \ldots, x_{n}$ цветами $\{1,2, \ldots, r\}$ слева направо $\chi\left(x_{1}\right)=1, \chi\left(x_{2}\right)=2, \ldots, \chi\left(x_{r}\right)=r, \chi\left(x_{r+1}\right)=1, \ldots$, $\chi\left(x_{n}\right)=1+(n-1) \bmod r$.

Очевидно, что раскраска $\chi$ уравновешена. Возьмем произвольную вершину $v \in \mathcal{V}$. Если $v$ - вершина одного из графов $R_{1} \cup R_{2}, R_{3} \cup R_{4}, \ldots, R_{t-1} \cup R_{t}$, то в соответствующем графе на расстоянии $\leqslant r$ от вершины $v$ имеются вершины всех $r$ цветов. Если $v-$ вершина одного из лучей $R_{t+1}, \ldots, R_{s}$, то $d(v, x)<r / 2$, а в графе $R_{1} \cup R_{2}$ на расстоянии $\leqslant r / 2$ от центра $x$ также имеются вершины всех $r$ цветов. Значит, индекс раскраски $\chi$ не превосходит $r$.

Если $t$ нечетно, раскрасим вершины графа $R_{1} \cup R_{2} \cup R_{3}$ согласно лемме 1 . Пусть $m-$ число вершин графра $R_{1} \cup R_{2} \cup R_{3}, m=r a+b, 0 \leqslant b<r$. Изменяя нумерацию цветов, можно считать, что цвета $\{1,2, \ldots, b\}$ использованы $a+1$ раз, а цвета $\{b+1, b+2, \ldots, r\}$ использованы $a$ раз. Начиная с цвета $(b+1) \bmod r$, продолжим эту раскраску на $\mathscr{V}$ так же, как и в случае четного $t$. Возьмем произвольную вершину $v \in \mathcal{V}$. Если $v$-вершина одного из графов $R_{1} \cup R_{2} \cup R_{3}, R_{4} \cup R_{5}, \ldots, R_{t-1} \cup R_{t}$, то в соответствующем графе на расстоянии $\leqslant r$ от вершины $v$ имеются вершины всех $r$ цветов. Если $v$ - вершина одного из лучей $R_{t+1}, \ldots, R_{s}$, то $d(v, x)<r / 2$, а на расстоянии $\leqslant r / 2$ от $x$ в графе $R_{1} \cup R_{2} \cup R_{3}$ также имеются вершины всех $r$ цветов. Следовательно, индекс раскраски не превосходит $r$.

Для натурального числа $r>1$ дерево, содержашее $\geqslant r$ вершин, назовем $r$-крuтuческим, если после удаления любого его ребра хотя бы одно из двух образовавшихся деревьев имеет $<r$ вершин. Напомним, что диаметром конечного связного графа назьвается максимальное расстояние между его вершинами. 
Лемма 3. В r-критическом дереве $G(\mathcal{V}, E)$ диаметра $d \geqslant r-1$ найдется вершина $x$, при удалении которой дерево распадается на поддеревья с числом вериин $<r$ в каждом из них.

ДокАЗАТЕЛЬСтво. Выберем вершины $v_{0}, v_{d}$ такие, что $d\left(v_{0}, v_{d}\right)=d$. Пусть $v_{0}$, $v_{1}, \ldots, v_{d}-$ кратчайший путь от $v_{0}$ к $v_{d}$. Для каждого $i \in\{1,2, \ldots, d\}$ обозначим через $T_{i}$ дерево с корнем $v_{i}$, которое образуется удалением из $G(\mathscr{V}, E)$ ребра $\left(v_{i-1}, v_{i}\right)$. Поскольку $d\left(v_{0}, v_{d}\right)=d$, дерево $T_{d}$ состоит из одной вершины $v_{d}$. Выберем минимальное число $m \in\{1,2, \ldots, d\}$ такое, что дерево $T_{m}$ имеет $<r$ вершин. Положим $x=v_{m-1}$. Для каждой вершины $y$, смежной с $x$, обозначим через $T(y)$ дерево с корнем $y$, полученное удалением ребра $(x, y)$. Если $y \notin\left\{v_{0}, v_{1}, \ldots, v_{d}\right\}$, то после удаления ребра $(x, y)$ мы получаем два дерева $T(x)$ и $T(y)$. Так как $T(x)$ содержит вершины $v_{0}, v_{1}, \ldots, v_{d}$ и $d \geqslant r-1$, то в силу $r$-критичности число вершин графа $T(y)$ меньше $r$. Это же утверждение верно и для $y \in\left\{v_{0}, v_{1}, \ldots, v_{d}\right\}$ в силу выбора числа $m$ и $r$-критичности.

Лемма 4. Для любого $r$-критического дерева $G(\mathscr{V}, E)$ существует уравновешенная $r$-раскраска индекса $\leqslant r$ множества вершин $\mathscr{V}$.

ДокАЗАТЕЛЬСтво. Если диаметр $d$ дерева $\leqslant r$, то любая $r$-раскраска множества вершин $\mathscr{V}$ имеет индекс $\leqslant r$. Для $d>r$ выберем вершину $x$, удовлетворяющую заключению леммы 3 . Обозначим через $y_{1}, y_{2}, \ldots, y_{s}$ все смежные с $x$ вершины дерева. Рассмотрим деревья $T\left(y_{1}\right), T\left(y_{2}\right), \ldots, T\left(y_{s}\right)$ с корнями $y_{1}, y_{2}, \ldots, y_{s}$, полученные удалением ребер $\left(y_{1}, x\right),\left(y_{2}, x\right), \ldots,\left(y_{s}, x\right)$. В каждом из них выберем по одной вершине $z_{i}$, наиболее удаленной от корней. Обозначим через $R_{1}, R_{2}, \ldots, R_{s}$ подграфы графа $G(\mathscr{V}, E)$, определяемые кратчайшими путями от $x$ к $z_{1}, z_{2}, \ldots, z_{s}$. Тогда граф $S=R_{1} \cup R_{2} \cup \cdots \cup R_{s}$ является звездой радиуса $\leqslant r-1$.

Предположим, что среди лучей звезды $S$ имеется хотя бы два луча длины $\geqslant r / 2$. По лемме 2 существует уравновешенная $r$-раскраска индекса $\leqslant r$ множества вершин звезды $S$. Продолжим ее произвольным образом до уравновешенной раскраски $\chi$ множества $\mathscr{V}$. Возьмем любую вершину $v \in \mathscr{V}, v \neq x$, и выберем поддерево $T\left(y_{i}\right)$, вершиной которого является $v$. Так как число вершин дерева $T\left(y_{i}\right)$ меньше $r$, то $B\left(z_{i}, r\right) \subseteq B(v, r)$. Поскольку в шаре $B\left(z_{i}, r\right)$ содержатся точки всех $r$ цветов звезды $S$, то раскраска $\chi$ имеет индекс $\leqslant r$.

Наконец, допустим, что только один луч звезды $S$, скажем $R_{1}$, имеет длину $\geqslant r / 2$. Среди лучей $R_{2}, \ldots, R_{s}$ выберем луч, скажем $R_{2}$, максимальной длины. Так как $d>r$, то графф $R_{1} \cup R_{2}$ имеет $\geqslant r$ вершин. Занумеруем их в порядке движения от конца луча $R_{2}$ к концу луча $R_{1}$ и продолжим эту нумерацию произвольным образом на все множество $\mathcal{V}$. Упорядоченную последовательность $x_{1}, x_{2}, \ldots, x_{n}$ вершин графа раскрасим по правилу $\chi\left(x_{i}\right)=1+(i-1) \bmod r$. Уравновешенность раскраски $\chi$ очевидна. Возьмем произвольную вершину $v \in \mathscr{V}$. По определению раскраски $\chi$ в шаре $B(v, n)$ найдутся $r$ разноцветных точек графа $R_{1} \cup R_{2}$. Значит, индекс раскраски $\leqslant r$.

Для подмножества $A$ множества вершин графа $G(\mathscr{V}, E)$ обозначим через $G[A]$ граф с множеством вершин $A$ и множеством ребер $E \cap(A \times A)$. Поскольку мы рассматриваем лишь неориентированные графы, $(u, v)$ и $(v, u)$ означают одно и то же ребро с концами $u, v \in \mathscr{V}$. 
Лемма 5. Пусть $G(\mathscr{V}, E)$ - конечный связный граф, $r$ - натуральное число и множсество вершин $\mathscr{V}$ разбито на подмнохсества $\mathscr{V}_{1}, \mathscr{V}_{2}, \ldots, \mathscr{V}_{k}$ так, что все графы $G\left[\mathscr{V}_{1}\right], G\left[\mathscr{V}_{2}\right], \ldots, G\left[\mathscr{V}_{k}\right]$ связны и допускают уравновешенные $r$-раскраски $\chi_{1}$, $\chi_{2}, \ldots, \chi_{k}$ индекса $\leqslant r$. Тогда существует уравновешенная $r$-раскраска $\chi$ индекса $\leqslant r$ множества $\mathscr{V}$.

ДоКАЗАТЕЛЬСТво. Пусть $\mathscr{V}_{i}=\left\{x_{i 1}, x_{i 2}, \ldots, x_{i m_{i}}\right\}$. Изменяя нумерацию цветов, можно считать, что $\chi_{i}\left(x_{i j}\right)=1+(j-1) \bmod r$ для всех $i \in\{1,2, \ldots, k\}, j \in\left\{1,2, \ldots, m_{i}\right\}$. Запишем вершины $\mathscr{V}$ в последовательность

$$
x_{11}, x_{12}, \ldots, x_{1 m_{1}}, x_{21}, x_{22}, \ldots, x_{2 m_{2}}, \ldots, x_{k 1}, x_{k 2}, \ldots, x_{k m_{k}}
$$

и для $i$-го члена $v$ этой последовательности положим $\chi(v)=1+(i-1) \bmod r$. Очевидно, что $\chi$ - искомая раскраска множества $\mathcal{V}$.

ДоКАЗАТЕЛЬСТВо ТЕОРЕмЫ 2. Для $r=1$ теорема очевидна. Предположим, что $r>1$. Заменяя граф его остовом, можно считать, что $G(\mathscr{V}, E)$ - дерево. Последовательным стиранием ребер разобьем множество $\mathscr{V}$ на подмножества $\mathscr{V}_{1}, \mathscr{V}_{2}, \ldots, \mathscr{V}_{k}$ так, что $G\left[\mathscr{V}_{1}\right], G\left[\mathscr{V}_{2}\right], \ldots, G\left[\mathscr{V}_{k}\right]-r$-критические деревья. Применим леммы 4,5 .

ДОКАЗАТЕЛЬСТВо ТЕОРЕМЫ 3. Возьмем произвольные смежные вершины $x, y$. По теореме Караганиса [1] существует биекция $f:\{1,2, \ldots, n\} \rightarrow \mathscr{V}$ такая, что $f(1)=x$, $f(n)=y$ и $d(f(i), f(i+1)) \leqslant 3$ для всех $i \in\{1,2, \ldots, n-1\}$. Для произвольной вершины $v \in \mathscr{V}$ положим $\chi(v)=1+(f(v)+1) \bmod r$. Тогда $\chi^{-1}(1), \chi^{-1}(2), \ldots, \chi^{-1}(r)-$ требуемое разбиение $\mathcal{V}$.

Завершим заметку некоторыми замечаниями и комментариями.

ЗАмечАниЕ 1. Сопоставление теорем 1, 2 приводит к вопросу: можно ли указать уравновешенное $r$-разбиение индекса $\leqslant r-1$ множества $\mathscr{V}$ вершин произвольного связного графа $G(\mathscr{V}, E),|\mathscr{V}|=n, r \leqslant n$ ? Следующий пример дает отрицательньй ответ.

Рассмотрим граф $G_{n}\left(\mathscr{V}_{n}, E_{n}\right), n \geqslant 2$, где

$$
\mathscr{V}_{n}=\left\{x_{1}, x_{2}, \ldots, x_{n}\right\}, \quad E_{n}=\left\{\left(x_{1}, x_{2}\right),\left(x_{1}, x_{3}\right), \ldots,\left(x_{1}, x_{n}\right)\right\} .
$$

Есть только две 2-раскраски $\chi_{1}, \chi_{2}$ множества $\mathscr{V}_{n}$ индекса 1 . А именно,

$$
\begin{aligned}
& \chi_{1}\left(x_{1}\right)=1, \quad \chi_{1}\left(x_{2}\right)=\chi_{1}\left(x_{3}\right)=\cdots=\chi_{1}\left(x_{n}\right)=2, \\
& \chi_{2}\left(x_{1}\right)=2, \quad \chi_{2}\left(x_{2}\right)=\chi_{2}\left(x_{3}\right)=\cdots=\chi_{2}\left(x_{n}\right)=1 .
\end{aligned}
$$

Если $n>3$, то эти раскраски неуравновешенны. Следовательно, для $n>3$ и $r=2$ нет уравновешенньх раскрасок индекса $r-1$ множества $\mathscr{V}_{n}$.

ЗАмечаниЕ 2. Пусть $G$ - конечная группа с единицей $e, S \subseteq G,|S|=r, S=S^{-1}$, $e \in S$. Рассмотрим граф Кэли Сау $(G)$ с множеством вершин $G$ и множеством ребер вида $(x, y), x \neq y, x y^{-1} \in S$. Применяя к каждой связной компоненте графа Сау $(G)$ теорему 2 , а затем лемму 5 , получаем следующее утверждение.

Существует уравновешенное разбиение группы $G=A_{1} \cup A_{2} \cup \cdots \cup A_{r}$ такое, что $G=S^{r} A_{i}$ для всех $i \in\{1,2, \ldots, r\}$.

Если $S$ - подгрупп грушы $G$, то указать такое разбиение очень просто: каждьй левый смежньй класс групшы $G$ по подгруппе $S$ раскрасим в цвета $\{1,2, \ldots, r\}$ и обозначим через $A_{1}, A_{2}, \ldots, A_{r}$ одноцветные подмножества. Поэтому теорему 2 можно рассматривать как формальный аналог теоремы Лагранжа для графов. 
ЗАмЕчАниЕ 3. В связи с теоремой 1 возникает вопрос, можно ли множество вершин конечного связного графа разбить на $r$ подмножеств индекса 1 при условии, что локальная степень каждой вершины достаточно большая? Напомним, что локальная степень вершины, это число ребер, выходящих из этой вершины. Уточним вопрос. Верно ли, что для каждого натурального числа $r$ найдется натуральное число $f(r)$ такое, что каждьй конечньй связньй граф с локальными степенями вершин $\geqslant f(r)$ можно раскрасить в $r$ цветов так, что каждый шар радиуса 1 содержит вершины всех $r$ цветов?

Числа $f(1), f(2)$ определяются теоремой $1 f(1)=f(2)=1$. Следуюший пример показывает, что число $f(3)$ не существует.

Для каждого натурального числа $m$ положим $X_{m}=\{1,2, \ldots, 3 m\}$ и обозначим через $Y_{m}$ семейство всех $m$-подмножеств множества $X_{m}$. Рассмотрим граф $G_{m}$ с множеством вершин $\mathscr{V}_{m}=X_{m} \cup Y_{m}$ и множеством ребер $E_{m}$, где $(x, y) \in E_{m}$ тогда и только тогда, когда $x \in X_{m}, y \in Y_{m}$ и $x \in y$. Заметим, что локальная степень каждой вершины графа $G_{m} \geqslant m$. Зафиксируем произвольную 3 -раскраску $\chi: \mathscr{V}_{m} \rightarrow\{1,2,3\}$. Поскольку $\left|X_{m}\right|=3 m$, найдется одноцветное $m$-подмножество у множества $X_{m}$. Значит, $|\chi(B(y, 1))|<3$.

ЗАмЕчАнИЕ 4. Конечньй связный граф называется однородным степени $k$, если локальная степень каждой его вершины равна $k$. Назовем однородньй граф $G(\mathscr{V}, E)$ степени $k$ калейдоскопическим, если существует раскраска $\chi: \mathscr{V} \rightarrow\{1,2, \ldots, k+1\}$ такая, что каждый шар $B(x, 1), x \in \mathscr{V}$, содержит точки всех $k+1$ цветов. Подробнее о калейдоскопических графах и их связях с кодами Хемминга см. [2].

\section{СПИСОК ЦИТИРОВАННОЙ ЛИТЕРАТУРЫ}

[1] Karaganis J. On the cube of graphs // Canad. Math. Bull. 1969. V. 11. P. 295-296.

[2] Protasova K. D. Kaleidoscopic graphs // Math. Stud. 2002. V. 18. P. 3-9.

г. Киев

E-mail: kseniya@profit.net.ua 